DOI: 10.17516/1997-1397-2021-14-2-159-175

УДК 517.9

\title{
On the Theory of $\psi$-Hilfer Nonlocal Cauchy Problem
}

\author{
Mohammed A. Almalahi* \\ Satish K. Panchal ${ }^{\dagger}$ \\ Department of Mathematics \\ Dr. Babasaheb Ambedkar Marathwada University \\ Aurangabad (M.S), India
}

Received 10.08.2020, received in revised form 10.09.2020, accepted 20.11.2020

\begin{abstract}
In this paper, we derive the representation formula of the solution for $\psi$-Hilfer fractional differential equation with constant coefficient in the form of Mittag-Leffler function by using Picard's successive approximation. Moreover, by using some properties of Mittag-Leffler function and fixed point theorems such as Banach and Schaefer, we introduce new results of some qualitative properties of solution such as existence and uniqueness. The generalized Gronwall inequality lemma is used in analyze $\mathrm{E}_{\alpha}$-Ulam-Hyers stability. Finally, one example to illustrate the obtained results.
\end{abstract}

Keywords: fractional differential equations, fractional derivatives, $\mathrm{E}_{\alpha}$-Ulam-Hyers stability, fixed point theorem.

Citation: M.A. Almalahi, S.K. Panchal, On the Theory of $\psi$-Hilfer Nonlocal Cauchy Problem, J. Sib. Fed. Univ. Math. Phys., 2021, 14(2), 159-175. DOI: 10.17516/1997-1397-2021-14-2-159-175.

\section{Introduction}

In recent years, the scientific community has been paying more attention to fractional calculus because it is an effective tool in modeling many phenomenaIn various fields of engineering and science, since its non-local properties are suitable for describing memory phenomena such as non-local elasticity, polymers, diffusion in complex medium, biological, electrochemical chemistry, porous media, viscosity, electromagnetism, etc. For more details, we refer the reader to monographs of Kilbas et al. [12], Samko et al. [21], Hilfer [10], Podlubny [19] and the papers [5,9]. In the recently years, Kilbas et al. in [12] introduced the properties of fractional integrals and fractional derivatives of a function with respect to another function. Sousa and Oliveira [22] proposed a $\psi$-Hilfer fractional operator and extended few previous works dealing with the Hilfer $[7,10]$. Moreover, they discussed some important qualitative properties of solutions such as existence, uniqueness, and stability results in the following papers $[18,22-24]$. Over the last years, the stability results of fractional differential equations have been robustly developed. Very significant contributions about this topic were introduced by Ulam [28], Hyers [11] and this type of stability is called Ulam-Hyers stability. Thereafter, the Ulam-Hyers stability was extended by Rassias [20] in 1978 to a new type of stability which called Ulam-Hyers-Rassias stability. For some recent results of stability analysis, we refer the reader to a series of papers $[2,3,14,17,18,24,26,30,31]$. For the existence and uniqueness results of different classes of initial value problem for fractional differential equations involving $\psi$-Hilfer derivative operator, one can see $[1-4,15,27]$. More recently, Wang and $\mathrm{Li}$ in [32] introduced four new types of $\mathrm{E}_{\alpha}$-Ulam stabilities. Gao et al., in [8] established the existence and uniqueness of solutions to the Hilfer nonlocal boundary value problem by using some properties of Hilfer fractional calculus, Mittag-Leffler functions, and fixed

\footnotetext{
*aboosama736242107@gmail.com https://orcid.org/0000-0001-5719-086X

†drpanchalsk@gmail.com

(c) Siberian Federal University. All rights reserved
} 
point methods. Kucche et al., in [13] obtained representation formula for the solution of Cauchy problem in the form of Mittag-Leffler function.

Motivated by $[8,13,32]$, in this paper, we use Picard's successive approximation technique to obtain representation formula for the solution of linear Cauchy problem with constant coefficient

$$
\begin{aligned}
{ }^{H} D_{a^{+}}^{\alpha, \beta ; \psi} y(t) & =\lambda y(t)+h(t), n-1<\alpha<n, \beta \in[0,1], t \in J:=(a, b], \\
y_{\psi}^{[n-j]} I_{a^{+}}^{n-\gamma ; \psi} y(a) & =c_{j}, j=1,2, \ldots, n, \quad \alpha \leqslant \gamma=\alpha+n \beta-\alpha \beta .
\end{aligned}
$$

in the form of Mittag-Leffler function, where $y_{\psi}^{[n-j]} y(t)=\left(\frac{1}{\psi^{\prime}(t)} \frac{d}{d t}\right)^{n-j} y(t), j=1,2, \ldots, n$. Furthermore, we introduce new results of some qualitative properties of solution such as existence, uniqueness, and $\mathrm{E}_{\alpha}$-stability results of a nonlinear $\psi$-Hilfer fractional differential equation

$$
\begin{gathered}
{ }^{H} D_{a^{+}}^{\alpha, \beta ; \psi} y(t)=\lambda y(t)+f(t, y(t)), \quad \alpha \in(1,2), \quad \beta \in[0,1], \quad t \in J:=(a, b], \\
y(a)=0, \quad y(b)=\sum_{i=1}^{m} \delta_{i} I_{a^{+}}^{\zeta, \psi} y\left(\tau_{i}\right), \quad \tau_{i} \in(a, b],
\end{gathered}
$$

where ${ }^{H} D_{a^{+}}^{\alpha, \beta, \psi}$ denotes the $\psi$-Hilfer fractional derivative of order $\alpha \in(1,2)$, type $\beta \in[0,1]$, $\gamma=\alpha+\beta(2-\alpha), \lambda<0, m \in \mathbb{N}$, and $f:(a, b] \times \mathbb{R} \longrightarrow \mathbb{R}$ is given function satisfying some assumptions that will be specified later.

To the best of our knowledge, this is the first paper dealing with $\psi$-Hilfer fractional derivative with constant coefficient of order $\alpha \in(1,2)$. In consequence, our findings of the present work will be a useful contribution to the existing literature on the topic.

This paper is organized as follows: In Section 2, we recall the basic definitions and prove some lemmas which are used throughout this paper, also we present the concepts of some fixed point theorems. In Section 3 , we derive representation formula for the solution of the problem (0.1)-(0.2) in the form of Mittag-Leffler function. Furthermore, we derive an equivalent fractional integral equation to the nonlocal problem (0.3)-(0.4). In Section 4, we study the existence and uniqueness results of $\psi$-Hilfer nonlocal problem $(0.3)-(0.4)$ by using some properties of Mittag-

Leffler function and fixed point theorems. In Section 5, we discuss $\mathrm{E}_{\alpha}$-Ulam-Hyers stability of solution to a given problem. In Section 6 we give one example to illustrate our results. Concluding remarks about our results in the last section.

\section{Preliminary}

Let $[a, b] \subset \mathbb{R}^{+}$with $0<a<b<\infty$. For $\gamma=\alpha+\beta(2-\alpha), 1<\alpha<2,0 \leqslant \beta \leqslant 1$. Then $1<\gamma \leqslant 2$. Let $\psi \in C^{1}[a, b]$ be an increasing function with $\psi^{\prime} \neq 0$, for all $t \in[a, b]$, the weighted space $C_{2-\gamma ; \psi}[a, b]$ of continuous function $f:[a, b] \rightarrow \mathbb{R}$ is defined by

$$
C_{2-\gamma ; \psi}[a, b]=\left\{f:(a, b] \rightarrow \mathbb{R} ;(\psi(t)-\psi(a))^{2-\gamma} f(t) \in C[a, b]\right\}, \quad 1<\gamma \leqslant 2 .
$$

Obviously $C_{2-\gamma ; \psi}[a, b]$ is the Banach spaces with the norm

$$
\|f\|_{C_{2}-\gamma ; \psi[a, b]}=\max _{t \in[a, b]}\left|(\psi(t)-\psi(a))^{2-\gamma} f(t)\right| .
$$

Next, define $L_{p}([a, b], \mathbb{R})$ the Banach space of all Lebesgue measurable functions $\mu:[a, b] \rightarrow \mathbb{R}$ with $\|\mu\|_{L_{p}[a, b]}<\infty$.

Definition 1.1 ([12]). Let $\alpha>0, f \in L_{1}[a, b]$. Then, the $\psi$-Riemann-Liouville fractional integral of a function $f$ with respect to $\psi$ is defined by

$$
I_{a^{+}}^{\alpha, \psi} f(t)=\frac{1}{\Gamma(\alpha)} \int_{a}^{t} \psi^{\prime}(s)(\psi(t)-\psi(s))^{\alpha-1} f(s) d s
$$


Definition 1.2 ([22]). Let $n-1<\alpha<n \in \mathbb{N}$, and $f, \psi \in C^{n}[a, b](-\infty<a<b<\infty)$ be two functions such that $\psi$ is increasing and $\psi^{\prime}(t) \neq 0$, for all $t \in[a, b]$. The left-sided $\psi$-Hilfer fractional derivative of a function $f$ of order $\alpha$ and type $0 \leqslant \beta \leqslant 1$ is defined by

$$
{ }^{H} D_{a^{+}}^{\alpha, \beta, \psi} f(t)=I^{\beta(n-\alpha) ; \psi}\left(\frac{1}{\psi^{\prime}(t)} \frac{d}{d t}\right)^{n} I_{a^{+}}^{(1-\beta)(n-\alpha), \psi} f(t) .
$$

Lemma $1.1([12])$. Let $\alpha, \gamma>0$. Then

(1) $I_{a^{+}}^{\alpha, \psi} I_{a^{+}}^{v, \psi} f(t)=I_{a^{+}}^{\alpha+v, \psi} f(t)$

(2) $I_{a^{+}}^{\alpha, \psi}(\psi(t)-\psi(a))^{\gamma-1}=\frac{\Gamma(\gamma)}{\Gamma(\alpha+\gamma)}(\psi(t)-\psi(a))^{\alpha+\gamma-1}$

(3) ${ }^{H} D_{a^{+}}^{\gamma, \psi}(\psi(t)-\psi(a))^{\gamma-1}=0$.

Lemma 1.2 ([22]). If $f \in C^{n}[a, b], n-1<\alpha<n$, and $0 \leqslant \beta \leqslant 1$, then

$$
I_{a^{+}}^{\alpha ; \psi H} D_{a^{+}}^{\alpha, \beta, \psi} f(t)=f(t)-\sum_{k=1}^{n} \frac{(\psi(t)-\psi(a))^{\gamma-k}}{\Gamma(\gamma-k+1)} f_{\psi}^{[n-k]} I_{a^{+}}^{(1-\beta)(n-\alpha) ; \psi} f(a),
$$

where $f_{\psi}^{[n-k]} f(t)=\left(\frac{1}{\psi^{\prime}(t)} \frac{d}{d t}\right)^{n-k} f(t)$.

Theorem 1.1 ([6]). (Banach fixed point theorem) Let $X$ be a Banach space, $K \subset X$ be closed, and $G: K \rightarrow K$ be a strict contraction, i.e., $\|G(x)-G(y)\| \leqslant L\|x-y\|$ for some $0<L<1$ and all $x, y \in K$. Then $G$ has a fixed point in $K$.

Remark 1.1. To simplify the notation and the proof of some results, we will introduce the following notation

$$
Q_{\psi}^{\gamma-2}(t, a)=(\psi(t)-\psi(a))^{\gamma-2} \quad \text { and } \quad \mathscr{N}_{\psi}^{\alpha-1}(t, s)=\psi^{\prime}(s)(\psi(t)-\psi(s))^{\alpha-1} .
$$

Lemma $1.3([29])$. Let $\alpha \in(1,2]$ and $\beta>0$ be arbitrary. The function $E_{\alpha}(\cdot), E_{\alpha, \alpha}(\cdot)$ and $E_{\alpha, \beta}(\cdot)$ are nonnegative, and for all $z<0$

$$
E_{\alpha}(z):=E_{\alpha, 1}(z) \leqslant 1, \quad E_{\alpha, \alpha}(z) \leqslant \frac{1}{\Gamma(\alpha)}, \quad E_{\alpha, \beta}(z) \leqslant \frac{1}{\Gamma(\beta)} .
$$

Moreover, for any $\lambda<0$ and $t_{1}, t_{2} \in[0,1]$, we have

$$
E_{\alpha, \alpha+\beta}\left(\lambda Q_{\psi}^{\alpha}\left(t_{2}, a\right)\right) \rightarrow E_{\alpha, \alpha+\beta}\left(\lambda Q_{\psi}^{\alpha}\left(t_{1}, a\right)\right) \text { as } t_{1} \rightarrow t_{2},
$$

where $E_{\alpha, \beta}$ is the Mittag-Leffler function.

Proof. See [29], Lemma 2 and [33].

Lemma 1.4. Let $\alpha>0, \beta>0, \gamma>0$ and $\lambda \in \mathbb{R}$. Then

$$
I_{a^{+}}^{\alpha, \psi} Q_{\psi}^{\beta-1}(t, a) E_{\gamma, \beta}\left(\lambda Q_{\psi}^{\gamma}(t, a)\right)=Q_{\psi}^{\alpha+\beta-1}(t, a) E_{\gamma, \alpha+\beta}\left(\lambda Q_{\psi}^{\gamma}(t, a) .\right.
$$

Proof. By Definition 1.1, we have

$$
\begin{aligned}
I_{a^{+}}^{\alpha, \psi} Q_{\psi}^{\beta-1}(t, a) E_{\gamma, \beta}\left(\lambda Q_{\psi}^{\gamma}(t, a)\right)= & \frac{1}{\Gamma(\alpha)} \int_{a}^{t} \mathscr{N}_{\psi}^{\alpha-1}(t, s) Q_{\psi}^{\beta-1}(s, a) E_{\gamma, \beta}\left(\lambda Q_{\psi}^{\gamma}(s, a)\right) d s \\
= & \frac{1}{\Gamma(\alpha)} \int_{a}^{t} \mathscr{N}_{\psi}^{\alpha-1}(t, s) Q_{\psi}^{\beta-1}(s, a) \sum_{n=0}^{\infty} \frac{\left(\lambda Q_{\psi}^{\gamma}(s, a)\right)^{n}}{\Gamma(\gamma n+\beta)} d s \\
& \quad-161-
\end{aligned}
$$




$$
=\sum_{n=0}^{\infty} \frac{\lambda^{n}}{\Gamma(\gamma n+\beta)} \frac{1}{\Gamma(\alpha)} \int_{a}^{t} \mathscr{N}_{\psi}^{\alpha-1}(t, s) Q_{\psi}^{\gamma n+\beta-1}(s, a) d s
$$

Via Lemma 1.1, we get

$$
I_{a^{+}}^{\alpha, \psi} Q_{\psi}^{\beta-1}(t, a) E_{\gamma, \beta}\left(\lambda Q_{\psi}^{\gamma}(t, a)\right)=Q_{\psi}^{\alpha+\beta-1}(t, a) E_{\gamma, \alpha+\beta}\left(\lambda Q_{\psi}^{\gamma}(t, a) .\right.
$$

Lemma 1.5. Let $\alpha>0, \beta>0, k>0, \lambda \in \mathbb{R}, z \in \mathbb{R}$ and $f \in C[a, b]$. Then

$$
I_{a^{+}}^{k, \psi} \int_{a}^{z} \mathscr{N}_{\psi}^{\alpha-1}(z, t) E_{\alpha, \alpha}\left(\lambda Q_{\psi}^{\alpha}(z, t)\right) f(t) d t=\int_{a}^{z}\left(\mathscr{N}_{\psi}^{\alpha+k-1}(z, t) E_{\alpha, \alpha+k}\left(\lambda Q_{\psi}^{\alpha}(z, t)\right) f(t) d t .\right.
$$

Proof. According to Definition 1.1 and Lemma 1.4, we obtain

$$
\begin{aligned}
& I_{a^{+}}^{k, \psi} \int_{a}^{z} \mathscr{N}_{\psi}^{\alpha-1}(z, t) E_{\alpha, \alpha}\left(\lambda Q_{\psi}^{\alpha}(z, t)\right) f(t) d t= \\
= & \frac{1}{\Gamma(k)} \int_{a}^{z} \mathscr{N}_{\psi}^{k-1}(z, u)\left\{\int_{a}^{u} \mathscr{N}_{\psi}^{\alpha-1}(u, t) E_{\alpha, \alpha}\left(\lambda Q_{\psi}^{\alpha}(u, t)\right) f(t) d t\right\} d u \\
= & \frac{1}{\Gamma(k)} \int_{a}^{z} \int_{t}^{z} \mathscr{N}_{\psi}^{\alpha-1}(u, t) E_{\alpha, \alpha}\left(\lambda Q_{\psi}^{\alpha}(u, t)\right) \mathscr{N}_{\psi}^{k-1}(z, u) f(t) d u d t \\
= & \frac{1}{\Gamma(k)} \int_{a}^{z} f(t) \Gamma(k) \mathscr{N}_{\psi}^{\alpha+k-1}(z, t) E_{\alpha, \alpha+k}\left(\lambda Q_{\psi}^{\alpha}(z, t)\right) d t \\
= & \int_{a}^{z}\left(\mathscr{N}_{\psi}^{\alpha+k-1}(z, t) E_{\alpha, \alpha+k}\left(\lambda Q_{\psi}^{\alpha}(z, t)\right) f(t) d t .\right.
\end{aligned}
$$

\section{Equivalent fractional integral equations}

In this section, we present explicit solutions to $\psi$-Hilfer fractional differential equations 0.1 , 0.2 in the form of Mittag-Leffler function. Moreover, we interduce equivalent fractional integral equation of the problem $0.3-0.4$.

Lemma 2.1. Let $h \in C_{n-\gamma ; \psi}(J, \mathbb{R}), \lambda \in \mathbb{R}, n-1<\alpha<n$ and $\beta \in[0,1]$. Then, the solution of Cauchy problem 0.1, 0.2 is given by

$$
y(t)=\sum_{j=1}^{n} c_{j} Q_{\psi}^{\gamma-j}(t, a) E_{\alpha, \gamma-j+1}\left[\lambda Q_{\psi}^{\alpha}(t, a)\right]+\int_{a}^{t} \mathscr{N}_{\psi}^{\alpha-1}(t, s) E_{\alpha, \alpha}\left[\lambda Q_{\psi}^{\alpha}(t, a)\right] h(s) d s .
$$

Proof. The equivalent fractional integral of the linear Cauchy problem (0.1)-(0.2) is

$$
y(t)=\sum_{j=1}^{n} \frac{Q_{\psi}^{\gamma-j}(t, a)}{\Gamma(\gamma-j+1)} c_{j}+\frac{\lambda}{\Gamma(\alpha)} \int_{a}^{t} \mathscr{N}_{\psi}^{\alpha-1}(t, s) y(s) d s+\frac{1}{\Gamma(\alpha)} \int_{a}^{t} \mathscr{N}_{\psi}^{\alpha-1}(t, s) h(s) d s .
$$

For explicit solutions of Eq. (2.2), we use the method of successive approximations, that is,

$$
\begin{aligned}
y_{0}(t) & =\sum_{j=1}^{n} \frac{Q_{\psi}^{\gamma-j}(t, a)}{\Gamma(\gamma-j+1)} c_{j}, \\
y_{k}(t) & =y_{0}(t)+\frac{\lambda}{\Gamma(\alpha)} \int_{a}^{t} \mathscr{N}_{\psi}^{\alpha-1}(t, s) y_{k-1}(t) d s+\frac{1}{\Gamma(\alpha)} \int_{a}^{t} \mathscr{N}_{\psi}^{\alpha-1}(t, s) h(s) d s .
\end{aligned}
$$


By Definition 1.1, Lemma 1.1 together with Eq. (2.3), we obtain

$$
\begin{aligned}
y_{1}(t) & =y_{0}(t)+\frac{\lambda}{\Gamma(\alpha)} \int_{a}^{t} \mathscr{N}_{\psi}^{\alpha-1}(t, s) y_{0}(s) d s+\int_{a}^{t} \mathscr{N}_{\psi}^{\alpha-1}(t, s) h(s) d s \\
& =\sum_{j=1}^{n} c_{j} \sum_{i=1}^{2} \frac{\lambda^{i-1} Q_{\psi}^{\alpha i+\beta(n-\alpha)-j}(t, a)}{\Gamma(\alpha i+\beta(n-\alpha)-j+1)}+\frac{1}{\Gamma(\alpha)} \int_{a}^{t} \mathscr{N}_{\psi}^{\alpha-1}(t, s) h(s) d s .
\end{aligned}
$$

Similarly, using Eqs. (2.3)-(2.5), we get

$$
y_{2}(t)=\sum_{j=1}^{n} c_{j} \sum_{i=1}^{3} \frac{\lambda^{i-1} Q_{\psi}^{\alpha i+\beta(n-\alpha)-j}(t, a)}{\Gamma(\alpha i+\beta(n-\alpha)-j+1)}+\int_{a}^{t} \sum_{i=1}^{2} \frac{\lambda^{i-1}}{\Gamma(\alpha i)} \psi^{\prime}(s) Q_{\psi}^{\alpha i-1}(t, s) h(s) d s .
$$

Continuing this process, the expression for $y_{k}(t)$ is given by

$$
y_{k}(t)=\sum_{j=1}^{n} c_{j} \sum_{i=1}^{k+1} \frac{\lambda^{i-1} Q_{\psi}^{\alpha i+\beta(n-\alpha)-j}(t, a)}{\Gamma(\alpha i+\beta(n-\alpha)-j+1)}+\int_{a}^{t} \sum_{i=1}^{k} \frac{\lambda^{i-1}}{\Gamma(\alpha i)} \psi^{\prime}(s) Q_{\psi}^{\alpha i-1}(t, s) h(s) d s .
$$

Taking the limit $k \rightarrow \infty$, we obtain the expression for $y_{k}(t)$, that is

$$
y(t)=\sum_{j=1}^{n} c_{j} \sum_{i=1}^{\infty} \frac{\lambda^{i-1} Q_{\psi}^{\alpha i+\beta(n-\alpha)-j}(t, a)}{\Gamma(\alpha i+\beta(n-\alpha)-j+1)}+\int_{a}^{t} \sum_{i=1}^{\infty} \frac{\lambda^{i-1}}{\Gamma(\alpha i)} \psi^{\prime}(s) Q_{\psi}^{\alpha i-1}(t, s) h(s) d s .
$$

Changing the summation index in the last expression, $i \rightarrow i+1$, we have

$$
y(t)=\sum_{j=1}^{n} c_{j} \sum_{i=0}^{\infty} \frac{\lambda^{i} Q_{\psi}^{\alpha i+\gamma-j}(t, a)}{\Gamma(\alpha i+\gamma-j+1)}+\int_{a}^{t} \sum_{i=0}^{\infty} \frac{\lambda^{i}}{\Gamma(\alpha i+\alpha)} \psi^{\prime}(s) Q_{\psi}^{\alpha i+\alpha-1}(t, s) h(s) d s .
$$

Using the definition of Mittag-Leffler function, we can obtain (2.1).

Lemma 2.2. Let $\gamma=\alpha+2 \beta-\alpha \beta$ such that $\alpha \in(1,2), \beta \in[0,1]$ and $f:(a, b] \times \mathbb{R} \rightarrow \mathbb{R}$ be a continuous function. Then $y$ is a solution of the problem (0.3)-(0.4) if and only if $y$ is a solution of the follwing integral equation

$$
\begin{array}{r}
y(t)=\frac{Q_{\psi}^{\gamma-1}(t, a) E_{\alpha, \gamma}\left(\lambda Q_{\psi}^{\alpha}(t, a)\right)}{K} \times\left[\sum_{i=1}^{m} \delta_{i} \int_{a}^{\tau_{i}} \mathscr{N}_{\psi}^{\alpha+\zeta-1}\left(\tau_{i}, s\right) E_{\alpha, \alpha+\zeta}\left(\lambda Q_{\psi}^{\alpha}\left(\tau_{i}, s\right)\right) f(s, y(s)) d s-\right. \\
\left.-\int_{a}^{b} \mathscr{N}_{\psi}^{\alpha-1}(b, s) E_{\alpha, \alpha}\left(\lambda Q_{\psi}^{\alpha}(b, s)\right) f(s, y(s)) d s\right]+ \\
+\int_{a}^{t} \mathscr{N}_{\psi}^{\alpha-1}(t, s) E_{\alpha, \alpha}\left(\lambda Q_{\psi}^{\alpha}(t, s)\right) f(s, y(s)) d s
\end{array}
$$

where

$$
K:=Q_{\psi}^{\gamma-1}(b, a) E_{\alpha, \gamma}\left(\lambda Q_{\psi}^{\alpha}(b, a)\right)-\sum_{i=1}^{m} \delta_{i} I_{a^{+}}^{\zeta, \psi} Q_{\psi}^{\gamma-1}\left(\tau_{i}, a\right) E_{\alpha, \gamma}\left(\lambda Q_{\psi}^{\alpha}\left(\tau_{i}, a\right)\right) \neq 0
$$

Proof. In view of Lemma 2.1, the problem $(0.3)-(0.4)$ is equivalent to

$$
\begin{aligned}
y(t)= & Q_{\psi}^{\gamma-1}(t, a) E_{\alpha, \gamma}\left(\lambda Q_{\psi}^{\alpha}(t, a)\right) c_{1}+Q_{\psi}^{\gamma-2}(t, a) E_{\alpha, \gamma-1}\left(\lambda Q_{\psi}^{\alpha}(t, a)\right) c_{2} \\
& +\int_{a}^{t} \mathscr{N}_{\psi}^{\alpha-1}(t, s) E_{\alpha, \alpha}\left(\lambda Q_{\psi}^{\alpha}(t, s)\right) f(s, y(s)) d s
\end{aligned}
$$


where

$$
c_{1}=\left(\frac{1}{\psi^{\prime}(t)} \frac{d}{d t}\right) I_{a^{+}}^{2-\gamma, \psi} y(a)=D_{a^{+}}^{\gamma-1, \psi} y(a) \quad \text { and } \quad c_{2}=I_{a^{+}}^{2-\gamma, \psi} y(a)
$$

By the first condition $(y(a)=0), \lim _{t \rightarrow a} Q_{\psi}^{\gamma-2}(t, a)=\infty$, we get $c_{2}=0$ and hence, Eq. (2.8) reduce to

$$
y(t)=Q_{\psi}^{\gamma-1}(t, a) E_{\alpha, \gamma}\left(\lambda Q_{\psi}^{\alpha}(t, a)\right) c_{1}+\int_{a}^{t} \mathscr{N}_{\psi}^{\alpha-1}(t, s) E_{\alpha, \alpha}\left(\lambda Q_{\psi}^{\alpha}(t, s)\right) f(s, y(s)) d s .
$$

Next, substitute $t=\tau_{i}$ into Eq. (2.9) and multiplying both side of Eq. (2.9) by $\delta_{i}$, we derive that

$$
\delta_{i} y\left(\tau_{i}\right)=\delta_{i} Q_{\psi}^{\gamma-1}\left(\tau_{i}, a\right) E_{\alpha, \gamma}\left(\lambda Q_{\psi}^{\alpha}\left(\tau_{i}, a\right)\right) c_{1}+\delta_{i} \int_{a}^{\tau_{i}} \mathscr{N}_{\psi}^{\alpha-1}\left(\tau_{i}, s\right) E_{\alpha, \alpha}\left(\lambda Q_{\psi}^{\alpha}\left(\tau_{i}, s\right)\right) f(s, y(s)) d s .
$$

Thus, we have

$$
\begin{aligned}
\sum_{i=1}^{m} \delta_{i} I_{a^{+}}^{\zeta, \psi} y\left(\tau_{i}\right)= & c_{1} \sum_{i=1}^{m} \delta_{i} I_{a^{+}}^{\zeta, \psi} Q_{\psi}^{\gamma-1}\left(\tau_{i}, a\right) E_{\alpha, \gamma}\left(\lambda Q_{\psi}^{\alpha}\left(\tau_{i}, a\right)\right) \\
& +\sum_{i=1}^{m} \delta_{i} I_{a^{+}}^{\zeta, \psi} \int_{a}^{\tau_{i}} \mathscr{N}_{\psi}^{\alpha-1}\left(\tau_{i}, s\right) E_{\alpha, \alpha}\left(\lambda Q_{\psi}^{\alpha}\left(\tau_{i}, s\right)\right) f(s, y(s)) d s .
\end{aligned}
$$

From Eqs. (2.9), (2.10) and second condition $\left(y(b)=\sum_{i=1}^{m} \delta_{i} I_{a^{+}}^{\zeta, \psi} y\left(\tau_{i}\right)\right)$, we get

$$
\begin{aligned}
c_{1}= & \frac{1}{K}\left[\sum_{i=1}^{m} \delta_{i} I_{a^{+}}^{\zeta, \psi} \int_{a}^{\tau_{i}} \mathscr{N}_{\psi}^{\alpha-1}\left(\tau_{i}, s\right) E_{\alpha, \alpha}\left(\lambda Q_{\psi}^{\alpha}\left(\tau_{i}, s\right)\right) f(s, y(s)) d s\right. \\
& \left.-\int_{a}^{b} \mathscr{N}_{\psi}^{\alpha-1}(b, s) E_{\alpha, \alpha}\left(\lambda Q_{\psi}^{\alpha}(b, s)\right) f(s, y(s)) d s\right] .
\end{aligned}
$$

Substitute Eq. (2.11) into Eq. (2.9) and using Lemma 1.5, we obtain Eq. (2.6).

Conversely, applying $D_{0^{+}}^{\gamma ; \psi}$ on both sides of Eq. (2.6) and using the fact $D_{a^{+}}^{\gamma, \psi} Q_{\psi}^{\gamma-1}(t, a)=0$, we can easily prove that

$$
{ }^{H} D_{a^{+}}^{\alpha, \beta ; \psi} y(t)=\lambda y(t)+f(t, y(t)) .
$$

Next, take $t \rightarrow a$ in Eq. (2.6), we get $y(a)=0$. On the other hand, applying $I_{a^{+}}^{\zeta, \psi}$ on both sides of Eq. (2.6) with taking $t \rightarrow \tau_{i}$, and multiply by $\delta_{i}$, we get

$$
\begin{aligned}
& \sum_{i=1}^{m} \delta_{i} I_{a^{+}}^{\zeta, \psi} y\left(\tau_{i}\right)= \frac{\sum_{i=1}^{m} \delta_{i} I_{a^{+}}^{\zeta, \psi} Q_{\psi}^{\gamma-1}\left(\tau_{i}, a\right) E_{\alpha, \gamma}\left(\lambda Q_{\psi}^{\alpha}\left(\tau_{i}, a\right)\right)}{K} \times \\
& \times\left[\sum_{i=1}^{m} \delta_{i} \int_{a}^{\tau_{i}} \mathscr{N}_{\psi}^{\alpha+\zeta-1}\left(\tau_{i}, s\right) E_{\alpha, \alpha+\zeta}\left(\lambda Q_{\psi}^{\alpha}\left(\tau_{i}, s\right)\right) f(s, y(s)) d s-\right. \\
&\left.\quad-\int_{a}^{b} \mathscr{N}_{\psi}^{\alpha-1}(b, s) E_{\alpha, \alpha}\left(\lambda Q_{\psi}^{\alpha}(b, s)\right) f(s, y(s)) d s\right]+ \\
& \quad+\sum_{i=1}^{m} \delta_{i} \int_{a}^{\tau_{i}} \mathscr{N}_{\psi}^{\alpha+\zeta-1}\left(\tau_{i}, s\right) E_{\alpha, \alpha+\zeta}\left(\lambda Q_{\psi}^{\alpha}\left(\tau_{i}, s\right)\right) f(s, y(s)) d s
\end{aligned}
$$

Thus, from Eq. (2.7), we can reduces Eq. (2.12) to 


$$
\begin{aligned}
\sum_{i=1}^{m} \delta_{i} I_{a^{+}}^{\zeta, \psi} y\left(\tau_{i}\right)=\frac{1}{K}( & \left.Q_{\psi}^{\gamma-1}(b, a) E_{\alpha, \gamma}\left(\lambda Q_{\psi}^{\alpha}(b, a)\right)-K\right) \times \\
\times & {\left[\sum_{i=1}^{m} \delta_{i} \int_{a}^{\tau_{i}} \mathscr{N}_{\psi}^{\alpha+\zeta-1}\left(\tau_{i}, s\right) E_{\alpha, \alpha+\zeta}\left(\lambda Q_{\psi}^{\alpha}\left(\tau_{i}, s\right)\right) f(s, y(s)) d s-\right.} \\
& \left.\quad-\int_{a}^{b} \mathscr{N}_{\psi}^{\alpha-1}(b, s) E_{\alpha, \alpha}\left(\lambda Q_{\psi}^{\alpha}(b, s)\right) f(s, y(s)) d s\right]+ \\
& +\sum_{i=1}^{m} \delta_{i} \int_{a}^{\tau_{i}} \mathscr{N}_{\psi}^{\alpha+\zeta-1}\left(\tau_{i}, s\right) E_{\alpha, \alpha+\zeta}\left(\lambda Q_{\psi}^{\alpha}\left(\tau_{i}, s\right)\right) f(s, y(s)) d s=y(b) .
\end{aligned}
$$

Thus, the nonlocal boundary conditions of the problem (0.3)-(0.4) are satisfied.

\section{Existence of solution}

The existence and uniqueness theorems of solutions to problem $(0.3)-(0.4)$ are presented in this section. For our analysis, the following assumptions should be valid.

$\left(H_{1}\right) f:(a, b] \times \mathbb{R} \rightarrow \mathbb{R}$ is jointly continuous.

$\left(H_{2}\right)$ There exist $0<q<1$ and a real function $\mathcal{V} \in L_{\frac{1}{q}}\left([a, b], \mathbb{R}^{+}\right)$such that $|f(t, y)| \leqslant \mathcal{V}(t)$ for all $t \in[a, b]$ and $y \in \mathbb{R}$.

$\left(H_{3}\right)$ There exist $0<q^{\prime}<1$ and a real function $\mathcal{W} \in L_{\frac{q^{\prime}}{}}\left([a, b], \mathbb{R}^{+}\right)$such that $|f(t, x)-f(t, y)| \leqslant$ $\mathcal{W}(t)|x-y|$ for all $t \in[a, b]$ and $x, y \in \mathbb{R}$.

For brevity, we set

$$
\begin{aligned}
& \rho=\left(\mathcal{B}\left(\frac{\alpha+\zeta-q^{\prime}}{1-q^{\prime}}, \frac{\gamma-q^{\prime}-1}{1-q^{\prime}}\right)\right)^{1-q^{\prime}} \sum_{i=1}^{m} \delta_{i} Q_{\psi}^{\alpha+\zeta-q^{\prime}+\gamma-2}\left(\tau_{i}, a\right), \\
& \sigma=\left(\frac{Q_{\psi}(b, a)}{\Gamma(\gamma) K}+1\right)\left(\mathcal{B}\left(\frac{\alpha-q^{\prime}}{1-q^{\prime}}, \frac{\gamma-q^{\prime}-1}{1-q^{\prime}}\right)\right)^{1-q^{\prime}} .
\end{aligned}
$$

Theorem 3.1. Assume that $f:(a, b] \times \mathbb{R} \rightarrow \mathbb{R}$ is continuous and satisfies $\left(H_{1}\right)-\left(H_{2}\right)$. Then the problem (0.3)-(0.4) has at least one solution in $C_{2-\gamma, \psi}[a, b]$.

Proof. Consider the operator $\mathcal{U}: C_{2-\gamma, \psi}[a, b] \longrightarrow C_{2-\gamma, \psi}[a, b]$ defined as

$$
\begin{array}{r}
\mathcal{U} y(t)=\frac{Q_{\psi}^{\gamma-1}(t, a) E_{\alpha, \gamma}\left(\lambda Q_{\psi}^{\alpha}(t, a)\right)}{K}\left[\sum_{i=1}^{m} \delta_{i} \int_{a}^{\tau_{i}} \mathscr{N}_{\psi}^{\alpha+\zeta-1}\left(\tau_{i}, s\right) E_{\alpha, \alpha+\zeta}\left(\lambda Q_{\psi}^{\alpha}\left(\tau_{i}, s\right)\right) f(s, y(s)) d s-\right. \\
\left.-\int_{a}^{b} \mathscr{N}_{\psi}^{\alpha-1}(b, s) E_{\alpha, \alpha}\left(\lambda Q_{\psi}^{\alpha}(b, s)\right) f(s, y(s)) d s\right]+ \\
\quad+\int_{a}^{t} \mathscr{N}_{\psi}^{\alpha-1}(t, s) E_{\alpha, \alpha}\left(\lambda Q_{\psi}^{\alpha}(t, s)\right) f(s, y(s)) d s
\end{array}
$$

It is obvious that the operator $\mathcal{U}$ is well defined. Define a bounded, closed, convex and nonempty set

$$
H_{\xi}=\left\{y \in C_{2-\gamma, \psi}[a, b]:\|y\|_{C_{2-\gamma, \psi}} \leqslant \xi\right\},
$$


of Banach space $C_{2-\gamma, \psi}[a, b]$ with

$$
\xi \geqslant\left\{\frac{Q_{\psi}(b, a)}{\Gamma(\gamma) K}[A+B]+C\right\}\|\mathcal{V}\|_{L_{\frac{1}{q}}[a, b]},
$$

where

$$
\begin{aligned}
A & :=\frac{1}{\Gamma(\alpha+\zeta)}\left(\frac{1-q}{\alpha+\zeta-q}\right)^{1-q} \sum_{i=1}^{m}\left|\delta_{i}\right| Q_{\psi}^{\alpha+\zeta-q}\left(\tau_{i}, a\right), \\
B & :=\frac{Q_{\psi}^{\alpha-q}(b, a)}{\Gamma(\alpha)}\left(\frac{1-q}{\alpha-q}\right)^{1-q} \\
C & :=\frac{Q_{\psi}^{2-\gamma+\alpha-q}(b, a)}{\Gamma(\alpha)}\left(\frac{1-q}{\alpha-q}\right)^{1-q} .
\end{aligned}
$$

Claim(1). The operator $\mathcal{U}$ is continuous in $H_{\xi}$. Consider a sequence $\left\{y_{n}\right\}_{n=1}^{\infty}$ such that $y_{n} \longrightarrow y$ in $C_{2-\gamma, \psi}[a, b]$. In view of Lemmas 1.3 and 1.5 , for $t \in(a, b]$, we have

$$
\begin{aligned}
& \left|Q_{\psi}^{2-\gamma}(t, a)\left[\mathcal{U} y_{n}(t)-\mathcal{U} y(t)\right]\right| \leqslant \frac{Q_{\psi}(t, a)}{\Gamma(\gamma) K}\left[\sum_{i=1}^{m} \frac{\left|\delta_{i}\right|}{\Gamma(\alpha+\zeta)} \int_{a}^{\tau_{i}} \mathscr{N}_{\psi}^{\alpha+\zeta-1}\left(\tau_{i}, s\right)\left|f_{y_{n}}-f_{y}\right| d s+\right. \\
& \left.+\frac{1}{\Gamma(\alpha)} \int_{a}^{b} \mathscr{N}_{\psi}^{\alpha-1}(b, s)\left|f_{y_{n}}-f_{y}\right| d s\right]+\frac{Q_{\psi}^{2-\gamma}(t, a)}{\Gamma(\alpha)} \int_{a}^{t} \mathscr{N}_{\psi}^{\alpha-1}(t, s)\left|f\left(s, y_{n}(s)\right)-f(s, y(s))\right| d s \leqslant \\
& \leqslant \frac{Q_{\psi}(t, a)}{\Gamma(\gamma) K}\left[\sum_{i=1}^{m} \frac{\left|\delta_{i}\right| \Gamma(\gamma-1)}{\Gamma(\alpha+\zeta+\gamma-1)} Q_{\psi}^{\alpha+\zeta+\gamma-2}\left(\tau_{i}, a\right)+\frac{\mathcal{B}(\alpha, \gamma-1) Q_{\psi}^{\alpha+\gamma-2}(b, a)}{\Gamma(\alpha)}\right] \times \\
& \quad \times\left\|f\left(\cdot, y_{n}(\cdot)\right)-f(\cdot, y(\cdot))\right\|_{2-\gamma, \psi}+\frac{\mathcal{B}(\alpha, \gamma-1) Q_{\psi}^{\alpha}(b, a)}{\Gamma(\alpha)}\left\|f\left(\cdot, y_{n}(\cdot)\right)-f(\cdot, y(\cdot))\right\|_{2-\gamma, \psi},
\end{aligned}
$$

where $\mathcal{B}(\alpha, \gamma-1)$ is Beta function. As $1<\gamma<2$, then $\frac{Q_{\psi}^{\gamma}\left(\tau_{i}, a\right)}{Q_{\psi}^{2}\left(\tau_{i}, a\right)}<1$, it follows that

$$
\begin{aligned}
& \left\|\mathcal{U} y-\mathcal{U} y_{n}\right\|_{C_{2-\gamma, \psi}} \leqslant \\
& \leqslant\left[\frac{Q_{\psi}(t, a)}{\Gamma(\gamma) K} \sum_{i=1}^{m} \frac{\left|\delta_{i}\right| \Gamma(\gamma-1)}{\Gamma(\alpha+\zeta+\gamma-1)} Q_{\psi}^{\alpha+\zeta}\left(\tau_{i}, a\right)+\left(\frac{Q_{\psi}(t, a)}{\Gamma(\gamma) K}+1\right) \frac{\mathcal{B}(\alpha, \gamma-1) Q_{\psi}^{\alpha}(b, a)}{\Gamma(\alpha)}\right] \times \\
& \quad \times\left\|f\left(\cdot, y_{n}(\cdot)\right)-f(\cdot, y(\cdot))\right\|_{2-\gamma, \psi} .
\end{aligned}
$$

Since $f$ is continuous function and $y_{n} \rightarrow y$ as $n \rightarrow \infty$, we have

$$
\left\|\mathcal{U} y-\mathcal{U} y_{n}\right\|_{C_{2-\gamma, \psi}} \rightarrow 0
$$

Thus, the operator $\mathcal{U}$ is continuous in $H_{\xi}$.

Claim(2). $\mathcal{U}$ maps bounded sets into bounded sets in $C_{2-\gamma, \psi}[a, b]$. For each $y \in H_{\xi}, t \in(a, b]$, by Lemmas 1.3, 1.5 and Hölder inequality, we have

$$
\begin{aligned}
& \left|Q_{\psi}^{2-\gamma}(t, a) \mathcal{U} y(t)\right| \leqslant \\
\leqslant & \frac{Q_{\psi}(t, a)}{\Gamma(\gamma) K}\left[\sum_{i=1}^{m} \frac{\left|\delta_{i}\right|}{\Gamma(\alpha+\zeta)} \int_{a}^{\tau_{i}} \mathscr{N}_{\psi}^{\alpha+\zeta-1}\left(\tau_{i}, s\right)|f(s, y(s))| d s\right. \\
& \left.+\frac{1}{\Gamma(\alpha)} \int_{a}^{b} \mathscr{N}_{\psi}^{\alpha-1}(b, s)|f(s, y(s))| d s\right]+\frac{Q_{\psi}^{2-\gamma}(t, a)}{\Gamma(\alpha)} \int_{a}^{t} \mathscr{N}_{\psi}^{\alpha-1}(t, s)|f(s, y(s))| d s
\end{aligned}
$$




$$
\begin{aligned}
& \leqslant \frac{Q_{\psi}(t, a)}{\Gamma(\gamma) K}\left[\sum_{i=1}^{m} \frac{\left|\delta_{i}\right|}{\Gamma(\alpha+\zeta)} \int_{a}^{\tau_{i}} \mathscr{N}_{\psi}^{\alpha+\zeta-1}\left(\tau_{i}, s\right) \mathcal{V}(s) d s+\frac{1}{\Gamma(\alpha)} \int_{a}^{b} \mathscr{N}_{\psi}^{\alpha-1}(b, s) \mathcal{V}(s) d s\right] \\
& +\frac{Q_{\psi}^{2-\gamma}(t, a)}{\Gamma(\alpha)} \int_{a}^{t} \mathscr{N}_{\psi}^{\alpha-1}(t, s) \mathcal{V}(s) d s \\
& \leqslant \frac{Q_{\psi}(t, a)}{\Gamma(\gamma) K}\left[\sum_{i=1}^{m} \frac{\left|\delta_{i}\right|}{\Gamma(\alpha+\zeta)}\left(\int_{a}^{\tau_{i}}\left(\mathscr{N}_{\psi}^{\alpha+\zeta-1}\left(\tau_{i}, s\right)\right)^{\frac{1}{1-q}} d s\right)^{1-q}\left(\int_{a}^{\tau_{i}}(\mathcal{V}(s))^{\frac{1}{q}} d s\right)^{q}\right. \\
& \left.+\frac{1}{\Gamma(\alpha)}\left(\int_{a}^{b}\left(\mathscr{N}_{\psi}^{\alpha-1}(b, s)\right)^{\frac{1}{1-q}} d s\right)^{1-q}\left(\int_{a}^{b}(\mathcal{V}(s))^{\frac{1}{q}} d s\right)^{q}\right] \\
& +\frac{Q_{\psi}^{2-\gamma}(t, a)}{\Gamma(\alpha)}\left(\int_{a}^{t}\left(\mathscr{N}_{\psi}^{\alpha-1}(t, s)\right)^{\frac{1}{1-q}} d s\right)^{1-q}\left(\int_{a}^{t}(\mathcal{V}(s))^{\frac{1}{q}} d s\right)^{q} \\
& \leqslant \frac{Q_{\psi}(t, a)}{\Gamma(\gamma) K}\left[\sum_{i=1}^{m} \frac{\left|\delta_{i}\right|}{\Gamma(\alpha+\zeta)}\left(\int_{a}^{\tau_{i}} \mathscr{N}_{\psi}^{\frac{\alpha+\zeta-1}{1-q}}\left(\tau_{i}, s\right) d s\right)^{1-q}\left(\int_{a}^{\tau_{i}}(\mathcal{V}(s))^{\frac{1}{q}} d s\right)^{q}\right. \\
& \left.+\frac{1}{\Gamma(\alpha)}\left(\int_{a}^{b} \mathscr{N}_{\psi}^{\frac{\alpha-1}{1-q}}(b, s) d s\right)^{1-q}\left(\int_{a}^{b}(\mathcal{V}(s))^{\frac{1}{q}} d s\right)^{q}\right] \\
& +\frac{Q_{\psi}^{1-\gamma}(t, a)}{\Gamma(\alpha)}\left(\int_{a}^{t} \mathscr{N}_{\psi}^{\frac{\alpha-1}{1-q}}(t, s) d s\right)^{1-q}\left(\int_{a}^{t}(\mathcal{V}(s))^{\frac{1}{q}} d s\right)^{q} \\
& \leqslant \frac{Q_{\psi}(t, a)}{\Gamma(\gamma) K}\left[\sum_{i=1}^{m} \frac{\left|\delta_{i}\right|}{\Gamma(\alpha+\zeta)}\left(\int_{a}^{\tau_{i}} \mathscr{N}_{\psi}^{\frac{\alpha+\zeta-q}{1-q}-1}\left(\tau_{i}, s\right) d s\right)^{1-q}\left(\int_{a}^{\tau_{i}}(\mathcal{V}(s))^{\frac{1}{q}} d s\right)^{q}\right. \\
& \left.+\frac{1}{\Gamma(\alpha)}\left(\int_{a}^{b} \mathscr{N}_{\psi}^{\frac{\alpha-q}{1-q}-1}(b, s) d s\right)^{1-q}\left(\int_{a}^{b}(\mathcal{V}(s))^{\frac{1}{q}} d s\right)^{q}\right] \\
& +\frac{Q_{\psi}^{2-\gamma}(t, a)}{\Gamma(\alpha)}\left(\int_{a}^{t} \mathscr{N}_{\psi}^{\frac{\alpha-q}{1-q}-1}(t, s) d s\right)^{1-q}\left(\int_{a}^{t}(\mathcal{V}(s))^{\frac{1}{q}} d s\right)^{q} \\
& \leqslant \frac{Q_{\psi}(t, a)}{\Gamma(\gamma) K}\left[\frac{1}{\Gamma(\alpha+\zeta)}\left(\frac{1-q}{\alpha+\zeta-q}\right)^{1-q} \sum_{i=1}^{m}\left|\delta_{i}\right| Q_{\psi}^{\alpha+\zeta-q}\left(\tau_{i}, a\right)\right. \\
& \left.+\frac{Q_{\psi}^{\alpha-q}(b, a)}{\Gamma(\alpha)}\left(\frac{1-q}{\alpha-q}\right)^{1-q}\right]\|\mathcal{V}\|_{L_{\frac{1}{q}}[a, b]}+\frac{Q_{\psi}^{2-\gamma+\alpha-q}(b, a)}{\Gamma(\alpha)}\left(\frac{1-q}{\alpha-q}\right)^{1-q}\|\mathcal{V}\|_{L_{\frac{1}{q}}[a, b]} \\
& \leqslant\left\{\frac{Q_{\psi}(b, a)}{\Gamma(\gamma) K}(A+B)+C\right\}\|\mathcal{V}\|_{L_{\frac{1}{q}}[a, b]} \leqslant \xi .
\end{aligned}
$$

Thus, $\mathcal{U}: H_{\xi} \longrightarrow H_{\xi}$, that is $\mathcal{U} H_{\xi}$ is uniformly bounded.

Claim(3). $\mathcal{U}$ maps bounded sets into equicontinuous set of $C_{2-\gamma, \psi}[a, b]$. For any $y \in H_{\xi}$, $t_{1}, t_{2} \in[a, b]$ such that $t_{1} \leqslant t_{2}$, using Lemmas 1.5 and 1.3 , we have

$$
\begin{aligned}
& \left|Q_{\psi}^{2-\gamma}\left(t_{2}, a\right) \mathcal{U} y\left(t_{2}\right)-Q_{\psi}^{2-\gamma}\left(t_{1}, a\right) \mathcal{U} y\left(t_{1}\right)\right| \leqslant \\
\leqslant & \left\|f_{y}\right\|_{C_{2-\gamma, \psi}}\left\{\frac{Q_{\psi}\left(t_{2}, a\right) E_{\alpha, \gamma}\left(\lambda Q_{\psi}^{\alpha}\left(t_{2}, a\right)\right)-Q_{\psi}\left(t_{1}, a\right) E_{\alpha, \gamma}\left(\lambda Q_{\psi}^{\alpha}\left(t_{1}, a\right)\right)}{|K|}\right\} \times \\
\times & {\left[\frac{\Gamma(\gamma-1)}{\Gamma(\alpha+\zeta+\gamma-1)} \sum_{i=1}^{m} \delta_{i} Q_{\psi}^{\alpha+\zeta+\gamma-1}\left(\tau_{i}, a\right)-\frac{\mathcal{B}(\alpha, \gamma-1)}{\Gamma(\alpha)} Q_{\psi}^{\alpha}(b, a)\right]+} \\
& +\frac{B(\alpha, \gamma-1)}{\Gamma(\alpha)}\left(Q_{\psi}^{\alpha}\left(t_{2}, a\right)-Q_{\psi}^{\alpha}\left(t_{1}, a\right)\right) \mid .
\end{aligned}
$$


By Eq. (1.1) as $t_{1} \longrightarrow t_{2}$, the right-hand side of the preceding inequality is not dependent on $y$ and goes to zero. Hence

$$
\left|Q_{\psi}^{2-\gamma}\left(t_{2}, a\right) \mathcal{U} y\left(t_{2}\right)-Q_{\psi}^{2-\gamma}\left(t_{1}, a\right) \mathcal{U} y\left(t_{1}\right)\right| \rightarrow 0, \quad \forall\left|t_{2}-t_{1}\right| \rightarrow 0, y \in H_{\xi}
$$

From the above claims, together with Arzela-Ascoli theorem, we infer that the operator $\mathcal{U}$ is completely continuous. In the remaining part of the proof, we only need to prove that the set

$$
\Delta=\left\{y \in C_{2-\gamma}[a, b]: y=\varpi \mathcal{U} y, \text { for some } \varpi \in(0,1)\right\}
$$

is bounded set. For each $t \in(a, b]$, let $y \in \Delta$, and $y=\varpi \mathcal{U} y$ for some $\varpi \in(0,1)$. Then $\|y\|_{2-\gamma, \psi} \leqslant\|\mathcal{U} y\|_{2-\gamma, \psi}$. Hence, by virtue of claim (2), we obtain

$$
\|y\|_{C_{2-\gamma, \psi}} \leqslant \xi .
$$

Thus, the set $\Delta$ is bounded. According to Schaefer's fixed point theorem we deduce that $\mathcal{U}$ has a fixed point which is a solution of the problem (0.3)-(0.4). The proof is completed.

Theorem 3.2. Assume that $\left(H_{1}\right)-\left(H_{3}\right)$ hold. If

$$
\left[\frac{Q_{\psi}(b, a)}{\Gamma(\gamma) K} \frac{\rho}{\Gamma(\alpha+\zeta)}+\frac{Q_{\psi}^{\alpha-q^{\prime}}(b, a) \sigma}{\Gamma(\alpha)}\right]\|\mathcal{W}\|_{L_{\frac{1}{q^{\prime}}}[a, b]}<1
$$

then the problem (0.3)-(0.4) has a unique solution in $C_{1-\gamma, \psi}[a, b]$.

Proof. In view of Theorem (3.1), we have known that the operator $\mathcal{U}$ defined by 3.1 is well defined and continuous. Now, we prove that $\mathcal{U}$ is a contraction map on $C_{2-\gamma, \psi}[a, b]$ with respect to the norm $\|\cdot\|_{C_{2-\gamma, \psi}}$. For each $y, y^{*} \in C_{2-\gamma, \psi}[a, b]$ and for all $t \in(a, b]$ with the help of Lemmas 1.3 , 1.5 and Hölder inequality, we have

$$
\begin{aligned}
\mid & Q_{\psi}^{2-\gamma}(t, a)\left[\mathcal{U} y(t)-\mathcal{U} y^{*}(t)\right]|\leqslant| \frac{Q_{\psi}(t, a) E_{\alpha, \gamma}\left(\lambda Q_{\psi}^{\alpha}(t, a)\right)}{K} \times \\
\times & {\left[\sum_{i=1}^{m} \delta_{i} \int_{a}^{\tau_{i}} \mathscr{N}_{\psi}^{\alpha+\zeta-1}\left(\tau_{i}, s\right) E_{\alpha, \alpha+\zeta}\left(\lambda Q_{\psi}^{\alpha}\left(\tau_{i}, s\right)\right)\left[f(s, y(s))-f\left(s, y^{*}(s)\right)\right] d s\right.} \\
& \left.-\int_{a}^{b} \mathscr{N}_{\psi}^{\alpha-1}(b, s) E_{\alpha, \alpha}\left(\lambda Q_{\psi}^{\alpha}(b, s)\right)\left[f(s, y(s))-f\left(s, y^{*}(s)\right)\right] d s\right] \\
+ & Q_{\psi}^{2-\gamma}(t, a) \int_{a}^{t} \mathscr{N}_{\psi}^{\alpha-1}(t, s) E_{\alpha, \alpha}\left(\lambda Q_{\psi}^{\alpha}(b, s)\right)\left[f(s, y(s))-f\left(s, y^{*}(s)\right)\right] d s \mid \leqslant \frac{Q_{\psi}(t, a)}{\Gamma(\gamma) K} \times \\
\times & {\left[\sum_{i=1}^{m} \frac{\left|\delta_{i}\right|}{\Gamma(\alpha+\zeta)}\left(\int_{a}^{\tau_{i}}\left(\mathcal{N}_{\psi}^{\alpha+\zeta-1}\left(\tau_{i}, s\right) Q_{\psi}^{\gamma-2}(s, a)\right)^{\frac{1}{1-q^{\prime}}} d s\right)^{1-q^{\prime}}\left(\int_{a}^{\tau_{i}}(\mathcal{W}(s))^{\frac{1}{q^{\prime}}} d s\right)^{q^{\prime}}\left\|y-y^{*}\right\|_{2-\gamma, \psi}\right.} \\
+ & \left.\frac{1}{\Gamma(\alpha)}\left(\int_{a}^{b}\left(\mathscr{N}_{\psi}^{\alpha-1}(b, s) Q_{\psi}^{\gamma-2}(s, a)\right)^{\frac{1}{1-q^{\prime}}} d s\right)^{1-q^{\prime}}\left(\int_{a}^{b}(\mathcal{W}(s))^{\frac{1}{q^{\prime}}} d s\right)^{q^{\prime}}\left\|y-y^{*}\right\|_{2-\gamma, \psi}\right] \\
+ & \frac{Q_{\psi}^{2-\gamma}(t, a)}{\Gamma(\alpha)}\left(\int_{a}^{t}\left(\mathcal{N}_{\psi}^{\alpha-1}(t, s) Q_{\psi}^{\gamma-2}(s, a)\right)^{\frac{1}{1-q^{\prime}}} d s\right)^{1-q^{\prime}}\left(\int_{a}^{t}(\mathcal{W}(s))^{\frac{1}{q^{\prime}}} d s\right)^{q^{\prime}}\left\|y-y^{*}\right\|_{2-\gamma, \psi} \leqslant \\
\leqslant & {\left[\frac { Q _ { \psi } ( t , a ) } { \Gamma ( \gamma ) K } \frac { 1 } { \Gamma ( \alpha + \zeta ) } \left(B\left(\frac{\alpha+\zeta-q^{\prime}}{1-q^{\prime}}, \frac{\gamma-q^{\prime}-1}{1-q^{\prime}}\right)^{1-q^{\prime}} \sum_{i=1}^{m} \delta_{i} Q_{\psi}^{\alpha+\zeta-q^{\prime}+\gamma-2}\left(\tau_{i}, a\right) \times\right.\right.}
\end{aligned}
$$




$$
\begin{gathered}
\left.\times\left(\frac{Q_{\psi}(t, a)}{\Gamma(\gamma) K}+1\right) \frac{Q_{\psi}^{\alpha-q^{\prime}}(b, a)}{\Gamma(\alpha)}\left(B\left(\frac{\alpha-q^{\prime}}{1-q^{\prime}}, \frac{\gamma-q^{\prime}-1}{1-q^{\prime}}\right)\right)^{1-q^{\prime}}\right]\|\mathcal{W}\|_{L_{q^{\prime}}[a, b]}\left\|y-y^{*}\right\|_{1-\gamma, \psi} \leqslant \\
\leqslant\left[\frac{Q_{\psi}(t, a)}{\Gamma(\gamma) K} \frac{\rho}{\Gamma(\alpha+\zeta)}+\frac{Q_{\psi}^{\alpha-q^{\prime}}(b, a) \sigma}{\Gamma(\alpha)}\right]\|\mathcal{W}\|_{L_{\frac{1}{q^{\prime}}}[a, b]}\left\|y-y^{*}\right\|_{2-\gamma, \psi}
\end{gathered}
$$

By (3.4), the operator $\mathcal{U}$ is a contraction map. According to Banach contraction principle, we conclude that the problem $(0.3)-(0.4)$ has a unique solution in $C_{2-\gamma, \psi}[a, b]$

\section{4. $\mathrm{E}_{\alpha}$-Ulam-Hyers stability}

In this section, we discuss the $\mathrm{E}_{\boldsymbol{\alpha}}$-Ulam-Hyers stability of the problem (0.3).

Lemma $4.1([23])$. Let $\alpha>0$ and $x, y$ be two nonnegative function locally integrable on $[a, b]$. Assume that $g$ is nonnegative and nondecreasing, and let $\psi \in C^{1}[a, b]$ an increasing function such that $\psi^{\prime}(t) \neq 0$ for all $t \in[a, b]$. If

$$
x(t) \leqslant y(t)+g(t) \int_{a}^{t} \mathscr{N}_{\psi}^{\alpha-1}(t, s) x(s) d s, \quad t \in[a, b],
$$

then

$$
x(t) \leqslant y(t)+\int_{a}^{t} \sum_{n=1}^{\infty} \frac{[g(t) \Gamma(\alpha)]^{n}}{\Gamma(n \alpha)} \mathscr{N}_{\psi}^{n \alpha-1}(t, s) y(s) d s, \quad t \in[a, b] .
$$

If $y$ be a nondecreasing function on $[a, b]$, then we have

$$
x(t) \leqslant y(t) E_{\alpha}\left\{g(t) \Gamma(\alpha) Q_{\psi}^{\alpha}(t, a)\right\}, \quad t \in[a, b] .
$$

Remark 4.1. A function $z \in C_{2-\gamma, \psi}[a, b]$ satisfies the inequality

$$
\left|{ }^{H} D_{a^{+}}^{\alpha, \beta, \psi} z(t)-\lambda z(t)-f(t, z(t))\right| \leqslant \varepsilon E_{\alpha} Q_{\psi}^{\alpha}(t, a), t \in(a, b],
$$

if and only if there exists a function $\eta \in C[a, b]$ such that

(i) $|\eta(t)| \leqslant \varepsilon E_{\alpha} Q_{\psi}^{\alpha}(t, a), \quad t \in[a, b]$;

(ii) $\left.{ }^{H} D_{a^{+}}^{\alpha, \beta, \psi} z(t)=\lambda z(t)+f(t, z(t))\right)+\eta(t), \quad t \in(a, b]$.

Definition 4.1 ([32]). The problem (0.3) is $E_{\alpha}$-Ulam-Hyers stable with respect to $E_{\alpha} Q_{\psi}^{\alpha}(t, a)$ if there exists $C_{E_{\alpha}}>0$ such that, for each $\varepsilon>0$ and each $z \in C_{2-\gamma, \psi}[a, b]$ satisfies the inequality (4.1), there exists a solution $x \in C_{2-\gamma, \psi}[a, b]$ of the problem (0.3) with

$$
\|z-x\|_{2-\gamma, \psi} \leqslant C_{E_{\alpha}} \varepsilon E_{\alpha}\left(\kappa Q_{\psi}^{\alpha}(t, a)\right), \quad t \in[a, b], \kappa>0 .
$$

Lemma 4.2. Let $1<\alpha<2,0 \leqslant \beta \leqslant 1$, if a function $z \in C_{2-\gamma, \psi}[a, b]$ satisfies the inequality (4.1), then $z$ satisfies the following integral inequality

$$
\begin{aligned}
& \left|z(t)-\mathcal{A}_{z}-\int_{a}^{t} \mathscr{N}_{\psi}^{\alpha-1}(t, s) E_{\alpha, \alpha}\left(\lambda Q_{\psi}^{\alpha}(t, s)\right) f(s, z(s)) d s\right| \leqslant \\
& \leqslant \varepsilon\left[\frac{1}{\Gamma(\gamma) K} \sum_{i=1}^{m}\left|\delta_{i}\right| Q_{\psi}^{\zeta}\left(\tau_{i}, a\right) E_{\alpha, \zeta+1} Q_{\psi}^{\alpha}\left(\tau_{i}, a\right)+\left(\frac{1}{\Gamma(\gamma) K}+1\right) E_{\alpha} Q_{\psi}^{\alpha}(b, a)\right],
\end{aligned}
$$


where

$$
\begin{aligned}
\mathcal{A}_{z}= & \frac{Q_{\psi}^{\gamma-1}(t, a) E_{\alpha, \gamma}\left(\lambda Q_{\psi}^{\alpha}(t, a)\right)}{K}\left[\sum_{i=1}^{m} \delta_{i} \int_{a}^{\tau_{i}} \mathscr{N}_{\psi}^{\alpha+\zeta-1}\left(\tau_{i}, s\right) E_{\alpha, \alpha+\zeta}\left(\lambda Q_{\psi}^{\alpha}\left(\tau_{i}, s\right)\right) f(s, z(s)) d s\right. \\
& \left.-\int_{a}^{b} \mathscr{N}_{\psi}^{\alpha-1}(b, s) E_{\alpha, \alpha}\left(\lambda Q_{\psi}^{\alpha}(b, s)\right) f(s, z(s)) d s\right] .
\end{aligned}
$$

Proof. Indeed by Remark 4.1, we have

$$
{ }^{H} D_{a^{+}}^{\alpha, \beta, \psi} z(t)=\lambda z(t)+f(t, z(t))+\eta(t), \quad t \in(a, b] .
$$

By Lemma 2.2, we obtain

$$
\begin{aligned}
z(t)= & \mathcal{A}_{z}-\frac{Q_{\psi}^{\gamma-1}(t, a) E_{\alpha, \gamma}\left(\lambda Q_{\psi}^{\alpha}(t, a)\right)}{K}\left[\sum_{i=1}^{m} \delta_{i} \int_{a}^{\tau_{i}} \mathscr{N}_{\psi}^{\alpha+\zeta-1}\left(\tau_{i}, s\right) E_{\alpha, \alpha+\zeta}\left(\lambda Q_{\psi}^{\alpha}\left(\tau_{i}, s\right)\right) \eta(s) d s\right. \\
& \left.-\int_{a}^{b} \mathscr{N}_{\psi}^{\alpha-1}(b, s) E_{\alpha, \alpha}\left(\lambda Q_{\psi}^{\alpha}(b, s)\right) \eta(s) d s\right]+\int_{a}^{t} \mathscr{N}_{\psi}^{\alpha-1}(t, s) E_{\alpha, \alpha}\left(\lambda Q_{\psi}^{\alpha}(t, s)\right) f(s, z(s)) d s \\
& +\int_{a}^{t} \mathscr{N}_{\psi}^{\alpha-1}(t, s) E_{\alpha, \alpha}\left(\lambda Q_{\psi}^{\alpha}(t, s)\right) \eta(s) d s .
\end{aligned}
$$

It follows from Lemma 1.3 and the fact $Q_{\psi}^{\gamma-1}(t, a)=\frac{Q_{\psi}^{\gamma}(t, a)}{Q_{\psi}(t, a)}<1$, that

$$
\begin{aligned}
& \left|z(t)-\mathcal{A}_{z}-\int_{a}^{t} \mathscr{N}_{\psi}^{\alpha-1}(t, s) E_{\alpha, \alpha}\left(\lambda Q_{\psi}^{\alpha}(t, s)\right) f(s, z(s)) d s\right| \leqslant \\
\leqslant & \frac{1}{\Gamma(\gamma) K}\left[\sum_{i=1}^{m} \frac{\left|\delta_{i}\right|}{\Gamma(\alpha+\zeta)} \int_{a}^{\tau_{i}} \mathscr{N}_{\psi}^{\alpha+\zeta-1}\left(\tau_{i}, s\right)|\eta(s)| d s+\frac{1}{\Gamma(\alpha)} \int_{a}^{b} \mathscr{N}_{\psi}^{\alpha-1}(b, s)|\eta(s)| d s\right] \\
& +\frac{1}{\Gamma(\alpha)} \int_{a}^{t} \mathscr{N}_{\psi}^{\alpha-1}(t, s)|\eta(s)| d s \leqslant \\
\leqslant & \frac{\varepsilon}{\Gamma(\gamma) K}\left[\sum_{i=1}^{m} \frac{\left|\delta_{i}\right|}{\Gamma(\alpha+\zeta)} \int_{a}^{\tau_{i}} \mathscr{N}_{\psi}^{\alpha+\zeta-1}\left(\tau_{i}, s\right) E_{\alpha} Q_{\psi}^{\alpha}(s, a) d s+\frac{1}{\Gamma(\alpha)} \int_{a}^{b} \mathscr{N}_{\psi}^{\alpha-1}(b, s) E_{\alpha} Q_{\psi}^{\alpha}(s, a) d s\right] \\
& +\frac{\varepsilon}{\Gamma(\alpha)} \int_{a}^{t} \mathscr{N}_{\psi}^{\alpha-1}(t, s) E_{\alpha} Q_{\psi}^{\alpha}(s, a) d s .
\end{aligned}
$$

By definition of Mittag-Leffler function and Theorem 1.1, we get

$$
\begin{aligned}
& \left|z(t)-\mathcal{A}_{z}-\int_{a}^{t} \mathscr{N}_{\psi}^{\alpha-1}(t, s) E_{\alpha, \alpha}\left(\lambda Q_{\psi}^{\alpha}(t, s)\right) f(s, z(s)) d s\right| \leqslant \\
& \leqslant \varepsilon\left[\frac{1}{\Gamma(\gamma) K} \sum_{i=1}^{m}\left|\delta_{i}\right| Q_{\psi}^{\zeta}\left(\tau_{i}, a\right) \sum_{n=0}^{\infty} \frac{Q_{\psi}^{\alpha(n+1)}\left(\tau_{i}, a\right)}{\Gamma((n+1) \alpha+\zeta+1)}+\left(\frac{1}{\Gamma(\gamma) K}+1\right) \sum_{n=0}^{\infty} \frac{Q_{\psi}^{\alpha(n+1)}(b, a)}{\Gamma((n+1) \alpha+1)}\right] \leqslant \\
& \leqslant \varepsilon\left[\frac{1}{\Gamma(\gamma) K} \sum_{i=1}^{m}\left|\delta_{i}\right| Q_{\psi}^{\zeta}\left(\tau_{i}, a\right) \sum_{n=0}^{\infty} \frac{Q_{\psi}^{\alpha n}\left(\tau_{i}, a\right)}{\Gamma(n \alpha+\zeta+1)}+\left(\frac{1}{\Gamma(\gamma) K}+1\right) \sum_{n=0}^{\infty} \frac{Q_{\psi}^{\alpha n}(b, a)}{\Gamma(n \alpha+1)}\right]= \\
& =\varepsilon\left[\frac{1}{\Gamma(\gamma) K} \sum_{i=1}^{m}\left|\delta_{i}\right| Q_{\psi}^{\zeta}\left(\tau_{i}, a\right) E_{\alpha, \zeta+1} Q_{\psi}^{\alpha}\left(\tau_{i}, a\right)+\left(\frac{1}{\Gamma(\gamma) K}+1\right) E_{\alpha} Q_{\psi}^{\alpha}(b, a)\right]
\end{aligned}
$$


In the forthcoming theorem, we prove the $\mathrm{E}_{\alpha}$-Ulam-Hyers stability result for the problem (0.3). For that, the following assumption should be valid.

$H_{4}$ There exist $L_{f}>0$ such that $|f(t, x)-f(t, y)| \leqslant L_{f}|x-y|$ for all $t \in[a, b]$ and $x, y \in \mathbb{R}$.

Theorem 4.1. Assume that $\left(H_{1}\right)$ and $\left(H_{4}\right)$, are satisfied. Then Eq. (0.3) is $E_{\alpha}$-Ulam-Hyers stable.

Proof. Let $\varepsilon>0, z \in C_{2-\gamma ; \psi}[a, b]$ be a function satisfying the inequality 4.1 and let $x \in$ $C_{2-\gamma ; \psi}[a, b]$ be the unique solution of the following problem

$$
\left\{\begin{array}{l}
{ }^{H} D_{a^{+}}^{\alpha, \beta ; \psi} x(t)=\lambda x(t)+f(t, x(t)), \quad t \in(a, b], \\
x\left(a^{+}\right)=z\left(a^{+}\right), \quad x(b)=z(b) .
\end{array}\right.
$$

Now, by using Lemma 2.2, we have

$$
x(t)=\mathcal{A}_{z}+\int_{a}^{t} \mathscr{N}_{\psi}^{\alpha-1}(t, s) E_{\alpha, \alpha} Q_{\psi}^{\alpha}(t, s) f(s, x(s)) d s, \quad t \in(a, b] .
$$

Hence, from $\left(\mathrm{H}_{4}\right)$ and Lemmas $4.2,1.3$, for each $t \in(a, b]$, we have

$$
\begin{aligned}
|z(t)-x(t)| \leqslant & \left|z(t)-\mathcal{A}_{z}-\int_{a}^{t} \mathscr{N}_{\psi}^{\alpha-1}(t, s) E_{\alpha, \alpha} Q_{\psi}^{\alpha}(t, s) f(s, z(s)) d s\right| \\
& +\left|\int_{a}^{t} \mathscr{N}_{\psi}^{\alpha-1}(t, s) E_{\alpha, \alpha} Q_{\psi}^{\alpha}(t, s)(f(s, z(s))-f(s, x(s))) d s\right| \leqslant \\
\leqslant & \varepsilon\left[\frac{1}{\Gamma(\gamma) K} \sum_{i=1}^{m}\left|\delta_{i}\right| Q_{\psi}^{\zeta}\left(\tau_{i}, a\right) E_{\alpha, \zeta+1} Q_{\psi}^{\alpha}\left(\tau_{i}, a\right)+\left(\frac{1}{\Gamma(\gamma) K}+1\right) E_{\alpha} Q_{\psi}^{\alpha}(b, a)\right] \\
& +\frac{L_{f}}{\Gamma(\alpha)} \int_{a}^{t} \mathscr{N}_{\psi}^{\alpha-1}(t, s)|z(t)-x(t)| d s .
\end{aligned}
$$

Using Lemma 4.1, we obtain

$$
\begin{aligned}
& |z(t)-x(t)| \leqslant \\
& \quad \leqslant \varepsilon\left[\frac{1}{\Gamma(\gamma) K} \sum_{i=1}^{m}\left|\delta_{i}\right| Q_{\psi}^{\zeta}\left(\tau_{i}, a\right) E_{\alpha, \zeta+1} Q_{\psi}^{\alpha}\left(\tau_{i}, a\right)+\left(\frac{1}{\Gamma(\gamma) K}+1\right) E_{\alpha} Q_{\psi}^{\alpha}(b, a)\right] E_{\alpha}\left(L_{f} Q_{\psi}^{\alpha}(t, a)\right) .
\end{aligned}
$$

For all $t \in(a, b]$, we have

$$
\begin{aligned}
\|z-x\|_{2-\gamma, \psi} \leqslant & \varepsilon\left[\frac{1}{\Gamma(\gamma) K} \sum_{i=1}^{m}\left|\delta_{i}\right| Q_{\psi}^{\zeta}\left(\tau_{i}, a\right) E_{\alpha, \zeta+1} Q_{\psi}^{\alpha}\left(\tau_{i}, a\right)+\left(\frac{1}{\Gamma(\gamma) K}+1\right) E_{\alpha} Q_{\psi}^{\alpha}(b, a)\right] \times \\
& \times Q_{\psi}^{\gamma-2}(t, a) E_{\alpha}\left(L_{f} Q_{\psi}^{\alpha}(t, a)\right) \\
\leqslant & \varepsilon\left[\frac{1}{\Gamma(\gamma) K} \sum_{i=1}^{m}\left|\delta_{i}\right| Q_{\psi}^{\zeta}\left(\tau_{i}, a\right) E_{\alpha, \zeta+1} Q_{\psi}^{\alpha}\left(\tau_{i}, a\right)+\left(\frac{1}{\Gamma(\gamma) K}+1\right) E_{\alpha} Q_{\psi}^{\alpha}(b, a)\right] \times \\
& \times Q_{\psi}^{\gamma-2}(b, a) E_{\alpha}\left(L_{f} Q_{\psi}^{\alpha}(t, a)\right) .
\end{aligned}
$$

Take $C_{E_{\alpha}}=\varepsilon\left[\frac{1}{\Gamma(\gamma) K} \sum_{i=1}^{m}\left|\delta_{i}\right| Q_{\psi}^{\zeta}\left(\tau_{i}, a\right) E_{\alpha, \zeta+1} Q_{\psi}^{\alpha}\left(\tau_{i}, a\right)+\left(\frac{1}{\Gamma(\gamma) K}+1\right) E_{\alpha} Q_{\psi}^{\alpha}(b, a)\right] Q_{\psi}^{\gamma-2}(b, a)$, we get

$$
\|z-x\|_{2-\gamma, \psi} \leqslant C_{E_{\alpha}} \varepsilon E_{\alpha}\left(L_{f} Q_{\psi}^{\alpha}(t, a)\right) .
$$

Thus, Eq. (0.3) is $\mathrm{E}_{\alpha}$-Ulam-Hyers stable. 


\section{An example}

In this section, one example is given to illustrate our theory results

Example 6.1 Consider the following problem

$$
\left\{\begin{array}{l}
{ }^{H} D_{0^{+}}^{\frac{3}{2}, \frac{2}{3} ; e^{t}} y(t)=-\frac{1}{2} y(t)+\frac{e^{-r t}}{1+e^{t}} \sin y(t), r>0 \quad t \in J:=(0,1], \\
y(0)=0, \quad y(1)=\frac{2}{3} I_{0^{+}}^{\frac{1}{2}} y\left(\frac{1}{2}\right) .
\end{array}\right.
$$

Here $\alpha=\frac{3}{2}, \beta=\frac{2}{3}, \gamma=\alpha+2 \beta-\alpha \beta=\frac{5}{6}, m=1, \tau_{1}=\frac{1}{2}, \delta_{1}=\frac{2}{3}, \zeta=\frac{1}{2},(a, b]=(0,1]$, $\psi(t)=e^{t}, \lambda=-\frac{1}{2}$ and $f(t, y(t))=\frac{e^{-r t}}{1+e^{t}} \sin y(t)$. Then

$$
|f(t, y(t))|=\left|\frac{e^{-r t}}{1+e^{t}} \sin y(t)\right| \leqslant\left|\frac{e^{-r t}}{1+e^{t}}\right| \leqslant \frac{e^{-r t}}{2}, \quad r>0 .
$$

Let $z, y \in \mathbb{R}$ and $t \in[0,1]$. Then

$$
|f(t, z(t))-f(t, y(t))| \leqslant\left|\frac{e^{-r t}}{1+e^{t}}(\sin z(t)-\sin y(t))\right| \leqslant \frac{e^{-r t}}{2}|z(t)-y(t)| .
$$

We note that $\mathcal{V}=\mathcal{W}=\frac{e^{-r t}}{2} \in L_{\frac{1}{q^{\prime}}}[0,1]$. Thus, for $t \in[0,1]$ and Choosing suitable $q^{\prime} \in(0,1)$, we can arrive at the following inequality

$$
\left[\frac{e-1}{\Gamma\left(\frac{5}{6}\right) K} \frac{\rho}{\Gamma(2)}+\frac{(e-1)^{\frac{3}{2}-q^{\prime}} \sigma}{\Gamma\left(\frac{3}{2}\right)}\right]\|\mathcal{W}\|_{L_{\frac{1}{q^{\prime}}}[0,1]}<1 .
$$

Then all the assumptions in Theorem 3.2 are satisfied, the problem (5.1) has a unique solution in $C_{\frac{7}{6}, e^{t}}[0,1]$. Also we see that the inequality

$$
\left|{ }^{H} D_{0^{+}}^{\frac{3}{2}, \frac{2}{3} ; e^{t}} y(t)+\frac{1}{2} y(t)-\frac{e^{-a t}}{1+e^{t}} \sin y(t)\right| \leqslant \varepsilon E_{\frac{3}{2}}\left(e^{t}-1\right)^{\frac{3}{2}}, \quad t \in[0,1]
$$

is satisfied. For $z, x \in C_{\frac{7}{6}}[0,1]$, we have

$$
\|z-x\|_{2-\gamma, \psi} \leqslant C_{E_{\frac{1}{3}}} \varepsilon E_{\frac{3}{2}}\left[\frac{1}{2}\left(e^{t}-1\right)^{\frac{1}{2}}\right], \quad t \in[0,1], \quad z, x \in C_{\frac{7}{6}}[0,1],
$$

where

$$
C_{E_{\frac{1}{3}}}=\left[\frac{1}{\Gamma\left(\frac{5}{6}\right)|K|} \frac{2}{3} E_{\frac{3}{2}, \frac{3}{2}}\left(e^{\frac{1}{2}}-1\right)^{\frac{3}{2}}+\left(\frac{1}{\Gamma\left(\frac{5}{6}\right) K}+1\right) E_{\frac{3}{2}}\left((e-1)^{\frac{3}{2}}\right)\right](e-1)^{\frac{5}{6}-2}>0 .
$$

Thus the Eq. (5.1) is $E_{\alpha}$-Ulam-Hyers stable.

\section{Concluding remarks}

We can conclude that the main results of this article have been successfully achieved, through some properties of Mittag-Leffler function and fixed point theorems such as Banach and Schaefer, we have investigated the existence and uniqueness of the solutions of nonlinear Cauchy problem for $\psi$-Hilfer fractional differential equation with constant coefficient. Further, we discussed $\mathrm{E}_{\alpha}$-Ulam-Hyers stability of solutions to such equations in the weighted space $C_{2-\gamma, \psi}[a, b]$. 


\section{References}

[1] M.S.Abdo, S.K.Panchal, Fractional integro-differential equations involving $\psi$-Hilfer fractional derivative, Advances in Applied Mathematics and Mechanics, 11(2019), 338-359 .

[2] M.A.Almalahi, S.K.Panchal, $\mathrm{E}_{\alpha}$-Ulam-Hyers stability result for $\psi$-Hilfer Nonlocal Fractional Differential Equation, Discontinuity, Nonlinearity, and Complexity, 2020. In press.

[3] M.A.Almalahi, M.S.Abdo, S.K.Panchal, Existence and Ulam-Hyers-Mittag-Leffler stability results of $\psi$-Hilfer nonlocal Cauchy problem, Rend. Circ. Mat. Palermo, II. Ser, (2020). DOI: $10.1007 / \mathrm{s} 12215-020-00484-8$

[4] M.A.Almalahi, M.S.Abdo, S.K.,Panchal, $\psi$-Hilfer Fractional functional differential equation by Picard operator method, Journal of Applied Nonlinear Dynamics, (2020).

DOI: $10.5890 /$ JAND.2020.12.011.

[5] A.Arara, M.Benchohra, N.Hamidi, J.J.Nieto, Fractional order differential equations on an unbounded domain, Nonlinear Anal. Theory Methods Appl., 72(2010), 580-586.

[6] K.Deimling, Nonlinear Functional Analysis, Springer, New York, 1985.

[7] K.M.Furati, M.D.Kassim, Existence and uniqueness for a problem involving Hilfer fractional derivative, Comput. Math. Applic, 64(2012), 1616-1626.

[8] Z.Gao, X.Yu, Existence results for BVP of a class of Hilfer fractional differential equations, Journal of Applied Mathematics and Computing, 56(2018), no. 1-2, 217-233.

[9] C.S.Goodrich, Existence of a positive solution to a class of fractional differential equations, Appl. Math. Lett., 23(2010), 1050-1055.

[10] R.Hilfer, Application of Fractional Calculus in Physics, World Scientific, Singapore, 1999.

[11] D.H.Hyers, G.Isac, Th.M.Rassias, Stability of Functional Equations in Several Variables, Progr. Nonlinear Differential Equations Appl., Birkh 646user, Boston, 34, 1998.

[12] A.A.Kilbas, H.M.Srivastava, J.J.Trujillo, Theory and Applications of Fractional Differential Equations, North-Holland Mathematics Studies, Vol. 207, Elsevier, Amsterdam, 2006.

[13] K.D.Kucche, A.D.Mali, J.Vanterler, C. da Sousa, On the Nonlinear $\psi$-Hilfer Fractional Differential Equations, Computational and Applied Mathematics, 38:73(2019).

DOI: $10.1007 / \mathrm{s} 40314-019-0833-5$.

[14] K.D.Kucche, A.D.Mali, Initial time difference quasilinearization method for fractional differential equations involving generalized Hilfer fractional derivative, Computational and Applied Mathematics, 39:31(2020). DOI: 10.1007/s40314-019-1004-4

[15] J.P.Kharade, K.D.Kucche, On the impulsive implicit $\psi$-Hilfer fractional differential equations with delay, Mathematical Methods in the Applied Sciences, 43(2020), no. 4, 1938-1952. DOI: $10.1002 /$ mma.6017

[16] K.Liu, J.Wang, D.O'Regan, Ulam-Hyers-Mittag-Leffler stability for $\psi$-Hilfer fractionalorder delay differential equations, Advances in Difference Equations, 50(2019), no. 1.

DOI: $10.1186 / \mathrm{s} 13662-019-1997-4$

[17] A.D.Mali, K.D.Kucche, Nonlocal Boundary Value Problem for Generalized Hilfer Implicit Fractional Differential Equations, arXiv preprint arXiv:2001.08479, 2020. 
[18] E.C.Oliveira, J.V.D.C.Sousa, Ulam-Hyers-Rassias stability for a class of fractional integrodifferential equations, Results Math., 73(2018), 111. DOI: 10.1007/s00025-018-0872-z

[19] I.Podlubny, Fractional Differential Equations: An Introduction to Fractional Derivatives, Fractional Differential Equations, to Methods of Their Solution and Some of Their Applications, Math. Sci. Eng., 198(1999), Elsevier, Amsterdam.

[20] T.M.Rassias, On the stability of the linear mapping in Banach spaces, Proc. Amer. Math. Soc., 72(1978), no. 2, 297-300.

[21] S.G.Samko, A.A.Kilbas, Marichev, O.I,:Fractional Integrals and Derivatives: Theory and Applications, Gordon and Breach, Yverdon, 1987.

[22] J.V.C.Sousa, E.C. de Oliveira, , On the $\psi$-Hilfer fractional derivative, Commun. Nonlinear Sci. Numer. Simul., 60(2018), 72-91. DOI: 10.1016/j.cnsns.2018.01.005

[23] J.Sousa, E.C. de Oliveira, A Gronwall inequality and the Cauchy-type problem by means of $\psi$-Hilfer operator, Differential equations and applications, 11(2019), no. 1, 87-106.

[24] J.V.D.C.Sousa, K.D.Kucche, E.C. De Oliveira, Stability of $\psi$-Hilfer impulsive fractional differential equations, Applied Mathematics Letters, 88(2019), 73-80.

[25] J.V.D.C.Sousa, E.C. de Oliveira, K.D.Kucche, On the fractional functional differential equation with abstract Volterra operator, Bulletin of the Brazilian Mathematical Society. New Series, 50(2019), no. 4, 803-822.

[26] J.V.D.C.Sousa, E.C. de Oliveira, On the Ulam-Hyers-Rassias stability for nonlinear fractional differential equations using the $\psi$-Hilfer operator, Journal of Fixed Point Theory and Applications, 20(2018), no. 3, 96.

[27] J.V.D.C.Sousa, K.D.Kucche, E.C. de Oliveira, On the Ulam-Hyers stabilities of the solutions of $\psi$-Hilfer fractional differential equation with abstract Volterra operator, Mathematical Methods in the Applied Sciences, 42(2019), no. 9, 3021-3032.

[28] S.M.Ulam, A Collection of Mathematical Problems, Interscience Tracts in Pure and Applied Mathematics, Vol. 8, Inter-science, New York-London, 1960.

[29] J.R.Wang, M.Feckan, Y.Zhou, Presentation of solutions of impulsive fractional Langevin equations and existence results, Eur. Phys. J. Spec. Top., 222(2013), 1857-1874,

[30] H.Wang, Existence of solutions for fractional anti-periodic BVP, Results Math., 88(2015), 227-245. DOI: $10.1007 / \mathrm{s} 00025-014-0431-1$

[31] J.Wang, Y.Zhang, Nonlocal initial value problems for differential equations with Hilfer fractional derivative, Appl. Math. Comput., 266(2015), 850-859.

DOI: $10.1016 /$ j.amc.2015.05.144

[32] J.Wang, X.Li, $\mathrm{E}_{\alpha}$-Ulam type stability of fractional order ordinary differential equations, Journal of Applied Mathematics and Computing, 45(2014), no. 1-2, 449-459.

[33] M.A.Almalahi, S.K.Panchal, Some existence and stability results for $\psi$-Hilfer fractional implicit diferential equation with periodic conditions, Journal of Mathematical Analysis and Modeling, 1(2020), no. 1, 1-19. 


\title{
К теории $\psi$-гильферовской нелокальной задачи Коши
}

\section{Мохаммед А. Альмалахи \\ Сатиш К. Панчал}

Кафедра математики Университет доктора Бабасахеба Амбедкара Маратвады

Аурангабад, Индия

\begin{abstract}
Аннотация. В данной статье мы выводим формулу представления решения дробнодифференциального уравнения $\psi$-Гильфера с постоянным коэффициентом в виде функции Миттаг-Леффлера с использованием последовательного приближения Пикара. Более того, используя некоторые свойства функции Миттаг-Леффлера и теоремы о неподвижной точке, такие как Банаха и Шефера, мы вводим новые результаты о некоторых качественных свойствах решения, таких как существование и единственность. Обобщенная лемма о неравенстве Гронуолла используется при анализе устойчивости $\mathrm{E}_{\alpha}$-Улама-Хайерса. Наконец, дан один пример, иллюстрирующий полученные результаты.
\end{abstract}

Ключевые слова: дробные дифференциальные уравнения, дробные производные, $\mathrm{E}_{\alpha}$-устойчивость Улама-Хайерса, теорема о неподвижной точке. 